\title{
Role of Tricuspid Valve Repair for Moderate Tricuspid Regurgitation during Minimally Invasive Mitral Valve Surgery
}

\author{
Bettina Pfannmueller ${ }^{1} \quad$ Alexander Verevkin ${ }^{1} \quad$ Michael Andrew Borger ${ }^{1}$ Meinhard Mende ${ }^{1}$ \\ Piroze Davierwala $^{1}$ Jens Garbade ${ }^{1}$ Friedrich Wilhelm Mohr ${ }^{1} \quad$ Martin Misfeld $^{1}$
} ${ }^{1}$ Department of Heart Surgery, Heart Center, University of Leipzig,
Leipzig, Germany

Thorac Cardiovasc Surg 2013;61:386-391.
Address for correspondence Bettina Pfannmueller, MD, Department of Heart Surgery, Heart Center, University of Leipzig, Strümpellstr. 39, Leipzig 04289, Germany (e-mail: pfab@med.uni-leipzig.de).

\begin{abstract}
Background The aim of this study was to investigate the impact of short- and midterm survival of tricuspid valve (TV) repair versus conservative therapy in patients with preoperative moderate functional tricuspid regurgitation (TR) undergoing minimally invasive mitral valve (MV) surgery.

Methods Between January 2002 and December 2009, a total of 430 patients with pure mitral regurgitation and concomitant moderate TR underwent minimally invasive MV surgery for mitral regurgitation at the Leipzig Heart Center without ( $n=336$; group A) and with ( $n=94$; group B) TV surgery. Mean age was $66.7 \pm 10.3$ years, mean LVEF was $58.0 \pm 13.8 \%$, and 206 patients $(47.9 \%$ ) were male. Average logEuroSCORE was $12.4 \pm 11.4 \%$. Follow-up was on average $4.6 \pm 2.4$ years and $97 \%$ completed.

Results Predischarge echocardiography showed no or mild TR in $51.1 \%$ of patients in group A versus $84.2 \%$ of patients in group B $(p<0.01)$. Overall 30-day mortality was $2.8 \%$ with no differences between both groups. Five-year survival was $82.9 \pm 4.1 \%$ for patients with TV repair versus $85.0 \pm 2.2 \%$ for patients without TV repair $(p=0.1)$ and it was $85.7 \pm 3.3 \%$ in patients with moderate and more postoperative TR versus $90.1 \pm 2.5 \%$ in patients with less than moderate postoperative TR $(p=0.08)$. Fiveyear freedom from TV-related reoperation was $98.8 \pm 0.7 \%$ for patients in group $A$ versus $98.9 \pm 0.1 \%$ for patients in group B $(p=0.8)$.

Keywords

- tricuspid valve

- mitral valve surgery

- minimally invasive surgery

Conclusions Patients undergoing MV surgery with moderate functional TR do not experience increased perioperative complication rates when a concomitant TV repair is performed. Our observations, combined with those of other groups, support current recommendations to perform concomitant TV repair in such patients, particularly if tricuspid annular dilation is present.
\end{abstract}

\section{Introduction}

The decision as to whether or not concomitant tricuspid valve (TV) surgery should be performed in patients with left-sided valve surgery was influenced for many years by an article from Braunwald et al in 1967. ${ }^{1}$ In this article, the authors concluded that tricuspid regurgitation (TR), in the presence of concomitant mitral valve (MV), would “... improve or disap-

received

October 4, 2012

accepted after revision

December 6, 2012

published online

March 8, 2013

pear after MV replacement and that TV replacement is seldom necessary."

However, several publications in the past 10 years have demonstrated that up to $74 \%$ of patients undergoing successful left-sided valve surgery will develop TR over time with poor outcomes. $^{2-5}$ In addition, TV-related reoperation is associated with an early mortality rate ranging between 0 and $37 \% .^{6-9}$

(c) 2013 Georg Thieme Verlog KG Stuttgart · New York
DOI http://dx.doi.org/ 10.1055/s-0033-1333844. ISSN 0171-6425. 
As a consequence of these more recent studies, American College of Cardiology/American Heart Association (ACC/AHA) guidelines for the management of patients with valvular disease give a level IIb $\mathrm{C}$ recommendation for concomitant TV surgery during MV operations: "Tricuspid annuloplasty may be considered for less than severe TR in patients undergoing MV surgery when there is pulmonary hypertension or tricuspid annular dilatation." ${ }^{10}$ The European guidelines give a level IIa $\mathrm{C}$ recommendation for "moderate secondary TR with dilated annulus $(>40 \mathrm{~mm})$ in a patient undergoing left-sided valve surgery." ${ }^{11}$

Despite these recommendations, there are currently no randomized trials and only a few retrospective studies that have compared postoperative outcome of patients with and without concomitant TV repair during left-sided valve surgery. The purpose of our study therefore was to compare medium-term outcomes in patients undergoing minimally invasive MV surgery in the presence of moderate functional TR with and without additional TV repair.

\section{Patients and Methods}

Between January 2002 and December 2009, a total of 2,183 patients with mitral regurgitation underwent minimally invasive MV surgery at the Leipzig Heart Center. Of these, 1,351 patients were men (61.9\%) and 832 were women (38.1\%). Of these, 231 patients underwent a concomitant TV repair, of which 109 were men ( $8.1 \%$ of the total men) and 122 were women (14.7\% of the total women; $p<0.001$ ).

Of the entire group of 2,183 patients, mitral regurgitation was accompanied by moderate TR in 430 patients, who formed the focus of this study. Patients with endocarditis were excluded. These patients were further divided into two groups. Group A consisted of patients undergoing isolated minimally invasive MV surgery $(n=336)$ and group B consisted of patients undergoing minimally invasive MV surgery and concomitant TV repair $(n=94)$. The decision as to whether or not the TV was repaired was left to the discretion of the operating surgeon. The number of patients undergoing additional TV surgery increased over the years of the study, reflecting a more aggressive approach to functional TR over time (see - Fig. 1).

Indications for MV surgery were symptomatic MR or asymptomatic MR in the presence of left ventricular dilation/dysfunction, pulmonary hypertension, and/or atrial fibrillation, in all patients. Pre-, intra-, and postoperative data were prospectively entered into a patient data management system and retrospectively analyzed. In addition, chart review and information from preoperative and predischarge echocardiographic reports were collected and analyzed. MR and TR were quantified by the measurement of the vena contracta in a four-chamber view. ${ }^{12,13}$

The minimally invasive technique for MV and TV surgery via a right anterolateral mini-thoracotomy has been described earlier by our group. ${ }^{14-17}$ The technique of MV repair/replacement and the decision for or against additional TV repair was at the surgeon's discretion, depending on the preoperative and/or intraoperative echocardiography data.

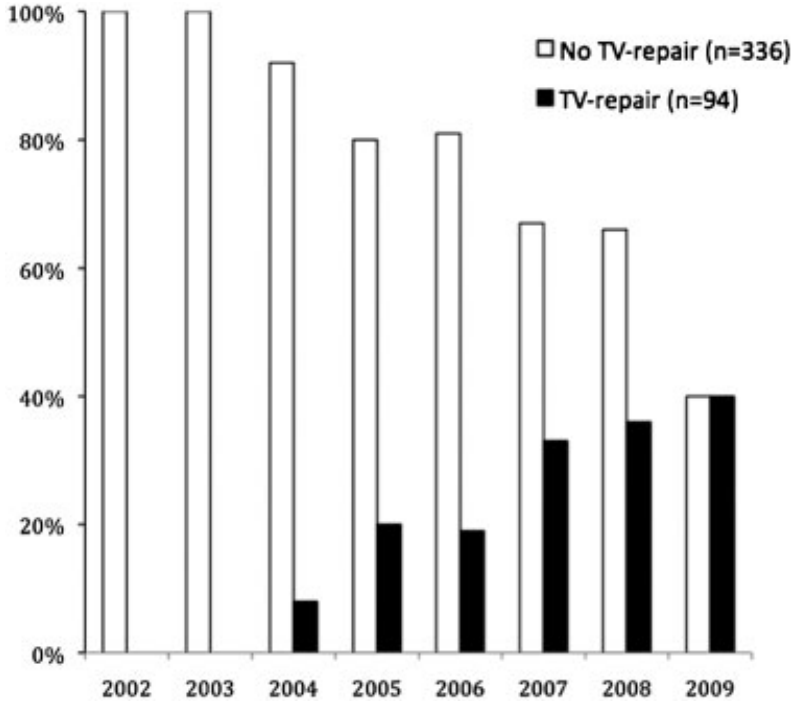

Fig. 1 Proportion of patients undergoing concomitant tricuspid valve (TV) repair over time.

The method of TV repair was also left to the surgeon's discretion and consisted of either a Kay suture annuloplasty ${ }^{18}$ or insertion of a semirigid Carpentier-Edwards Classic ring (Edwards Lifesciences, Irvine, CA, United States) or a flexible Cosgrove-Edwards annuloplasty band (Edwards Lifesciences). Atrial ablation procedure was performed in patients with symptomatic atrial fibrillation, which is recommended as IIa classification. ${ }^{19}$

Ethical approval was granted from the local Ethics Committee for this investigation.

\section{Follow-Up}

All patients were contacted by mail and were asked to answer a specific questionnaire on an annual basis. Patients who did not respond were contacted directly by telephone. If no further information was available, family physicians and/or referring cardiologists were contacted. Follow-up was 97\% complete with a mean follow-up time of $4.6 \pm 2.4$ years (range 0 to 9.8 years).

\section{Statistical Evaluation}

Results are displayed in the standard format with continuous variables expressed as mean \pm standard deviation and categorical data as proportions throughout the manuscript. Risk analysis was done with binary logistic regression analysis. Cumulative survival, as well as freedom from valve-related reoperation, was calculated by Kaplan-Meier methods. Differences in follow-up were calculated with 95\% confidence limits and compared by log-rank (Mantel Cox) test.

All the statistical analyses were performed using SPSS statistical package 17.0 (SPSS Corp., Birmingham, AL, United States). A $p$-value less than 0.05 was considered to be statistically significant.

\section{Results}

A total of 430 patients underwent minimally invasive MV surgery with $(n=336)$ or without $(n=94)$ concomitant TV 
Table 1 Preoperative patient variables

\begin{tabular}{|l|l|l|l|l|}
\hline & $\begin{array}{l}\text { All patients } \\
(\boldsymbol{n}=\mathbf{4 3 0})\end{array}$ & $\begin{array}{l}\text { MV surgery } \\
(\boldsymbol{n}=\mathbf{3 3 6})\end{array}$ & $\begin{array}{l}\text { MV surgery + TV repair } \\
(\boldsymbol{n}=\mathbf{9 4})\end{array}$ & $p$-Value \\
\hline Age (years) & $66.7 \pm 10.3$ & $66.5 \pm 10.4$ & $67.2 \pm 10.0$ & 0.6 \\
\hline Male & $206(47.9 \%)$ & $159(47.3 \%)$ & $47(50.0 \%)$ & 0.7 \\
\hline Preoperative NYHA & $2.6 \pm 0.7$ & $2.6 \pm 0.7$ & $2.6 \pm 0.7$ & 0.7 \\
\hline logEuroSCORE & $12.4 \pm 11.4 \%$ & $7.7 \pm 8.0 \%$ & $7.5 \pm 9.3 \%$ & 0.9 \\
\hline LVEF & $58.0 \pm 13.8 \%$ & $57.8 \pm 14.0 \%$ & $58.4 \pm 13.1 \%$ & 0.7 \\
\hline Pulmonary artery pressure & $48.6 \pm 14.3 \%$ & $49.4 \pm 14.5 \%$ & $46.0 \pm 13.3 \%$ & 0.5 \\
\hline Previous cardiac surgery & $31(7.2 \%)$ & $28(8.3 \%)$ & $3(3.2 \%)$ & 0.09 \\
\hline Atrial fibrillation & $205(47.7 \%)$ & $145(43.2 \%)$ & $60(63.8 \%)$ & 0.004 \\
\hline Diabetes mellitus & $73(17.1 \%)$ & $60(18.0 \%)$ & $13(14.0 \%)$ & 0.07 \\
\hline Permanent pacemaker & $37(8.6 \%)$ & $29(8.7 \%)$ & $8(8.5 \%)$ & 0.4 \\
\hline
\end{tabular}

Abbreviations: logEuroSCORE, logistic EuroSCORE-predicted risk of operative mortality; LVEF, left ventricular ejection fraction; MV, mitral valve; NYHA, New York Heart Association; TV, tricuspid valve.

repair due to moderate functional TR. The mean age of all patients was 66.7 years and nearly half were male (see -Table 1). Average New York Heart Association (NYHA) class was $2.6 \pm 0.7$. Left ventricular function was normal in the majority of patients and atrial fibrillation was present in almost half of the cohort. In 31 patients (7.2\%), a previous cardiac operation had been performed.

- Table 1 compares the demographic data of patients who did and did not undergo concomitant TV repair. The only significant difference between groups was the presence of atrial fibrillation, which was significantly more frequent in patients who underwent additional TV repair. Preoperative NYHA classification III to IV was present in $62.3 \%$ (259 out of 416) of all patients and there was no significant difference between the two groups ( $p=0.5$ ).

Operation, cross-clamp, and cardiopulmonary bypass (CPB) times were significantly shorter in patients without additional TV repair (operation time: $168 \pm 54 \mathrm{~min}$ vs. $221 \pm 54 \mathrm{~min}, p<0.001$; cross-clamp time: $71 \pm 35 \mathrm{~min}$ vs. $100 \pm 29 \min , p<0.001$; СРВ time: $124 \pm 43 \mathrm{~min}$ vs. $174 \pm 41 \mathrm{~min}$, all $p<0.001)$.

MV repair was performed in 420 patients (97.7\%), of which 327 patients (97.3\%) were in group A and 93 patients (98.9\%) were in group $B(p=0.3)$. The remainder of patients in both groups underwent MV replacement surgery.

TV repair in group B was performed using a Kay suture annuloplasty in 2 patients (2.1\%), a rigid Carpentier-Edwards ring in 40 patients (42.6\%), and a flexible Cosgrove-Edwards annuloplasty band in 52 patients (55.3\%).

Additional procedures were closure of an atrial septal defect in 47 patients (10.9\%) and an atrial ablation procedure in 186 patients (43.3\%). There was a significant difference in the incidence of atrial ablation procedures in both groups: group A, 130 out of 336 patients (38.7\%); group B, 56 out of 94 patients (59.6\%); $p<0.01$, which was related to a significant difference in the incidence of preoperative atrial fibrillation between both groups.

Predischarge echocardiography showed no or mild TR in $51.1 \%$ of patients in group A, and no or mild residual TR in
$84.2 \%$ of patients in group B $(p<0.01)$. Detailed echocardiographic data are displayed in $\mathbf{- F i g . ~} \mathbf{2}$

New postoperative permanent neurological deficits were detected in four patients $(0.9 \%)$, all of them were in group $\mathrm{A}$ $(p=0.8)$.

Postoperative rethoracotomy due to bleeding complications was necessary in 29 out of 336 patients (8.6\%) of group A and 11 out of 94 patients (11.7\%) of group B.

Overall, 30-day mortality was $2.8 \%$ with nine deaths (2.7\%) occurring in group A and three deaths (3.2\%) occurring in group B $(p=0.7)$.

-Fig. 3 displays mid-term survival of group A and B patients. Survival was $84.3 \pm 2.0 \% 5$ years postoperatively for the entire patient cohort, with $85.0 \pm 2.2 \% 5$-year survival in group A compared with $82.9 \pm 4.1 \%$ in group B (log-rank $p=0.1)$. - Fig. 4 depicts mid-term survival of patients with

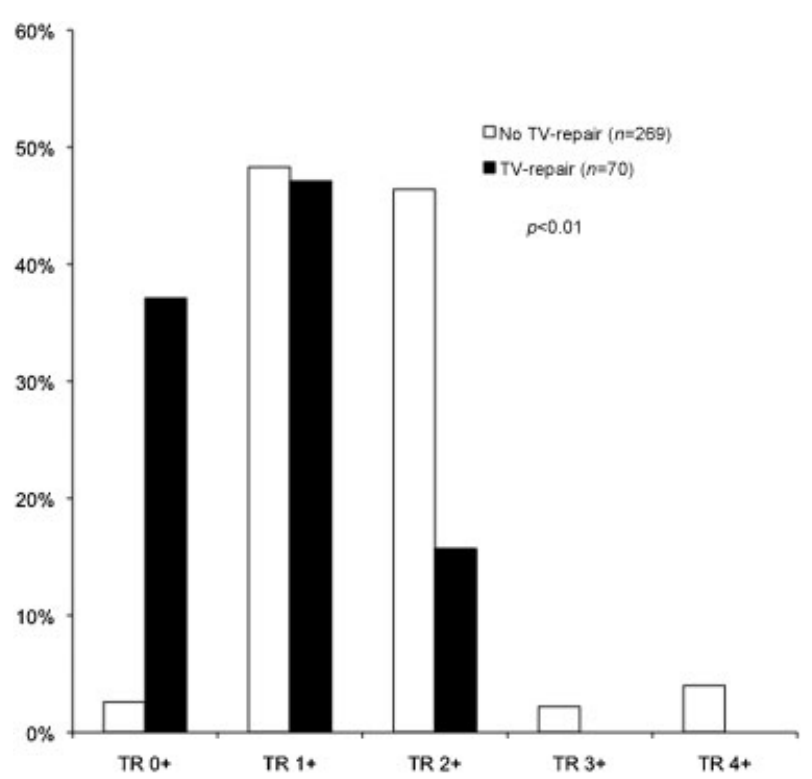

Fig. 2 Tricuspid regurgitation (TR) grade on predischarge echocardiography. 


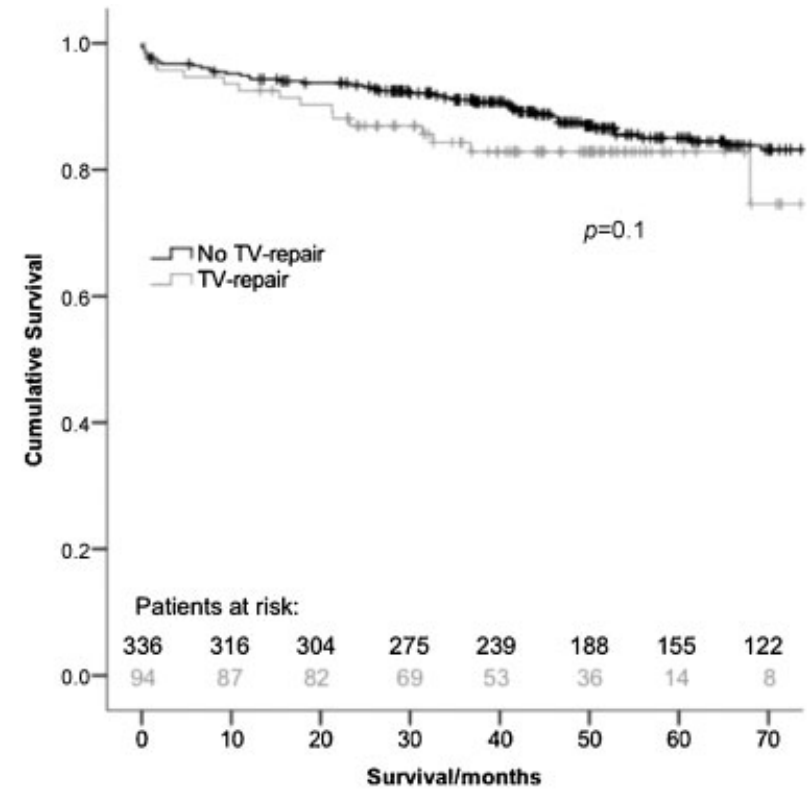

Fig. 3 Kaplan-Meier estimated survival for patients who did and did not undergo concomitant tricuspid valve (TV) repair.

residual $\mathrm{TR} \geq$ grade $2+$ versus patients with residual $\mathrm{TR}<$ grade $2+$. Five-year survival was $85.7 \pm 3.3 \%$ in the patient with moderate and more postoperative TR versus $90.1 \pm 2.5 \%$ for patients with less than moderate residual TR $(p=0.08)$.

At follow-up, NYHA classification I to II was present in $72.1 \%$ (178 out of 247 ) of patients, with no significant difference between the groups $(p=0.4)$.

Mid-term freedom from TV-related reoperation is displayed in - Fig. 5. After 5 years, freedom from TV-related reoperation was $98.8 \pm 0.4 \%$ for all patients, with $98.8 \pm 0.7 \%$ for patients in group $A$ and $98.9 \pm 0.1 \%$ for patients in group $B$

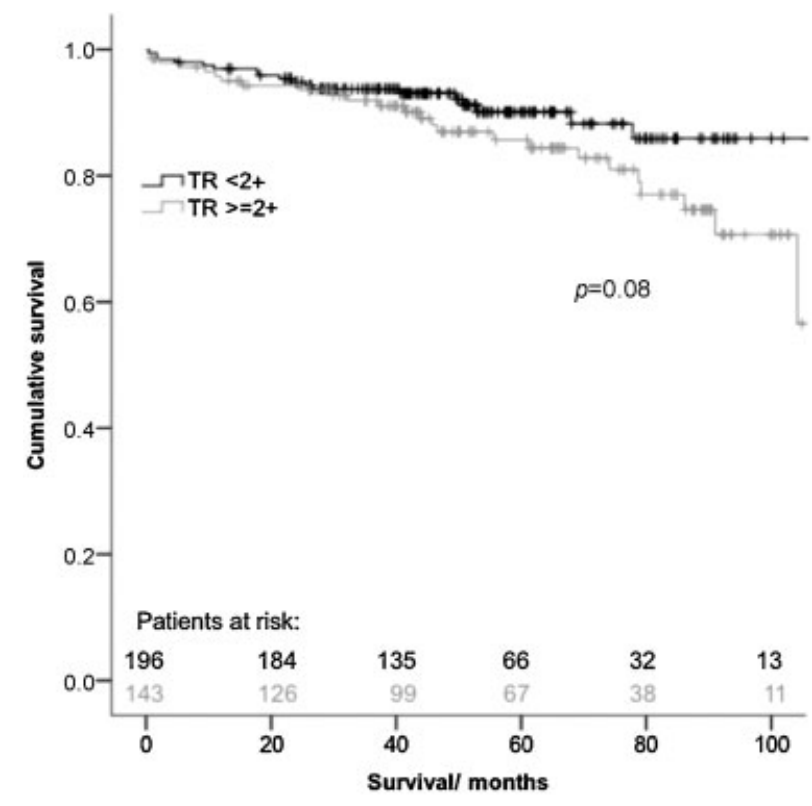

Fig. 4 Kaplan-Meier estimated survival for patients with residual tricuspid regurgitation (TR) grade $\geq 2+$ versus patients with residual TR grade $<2+$.

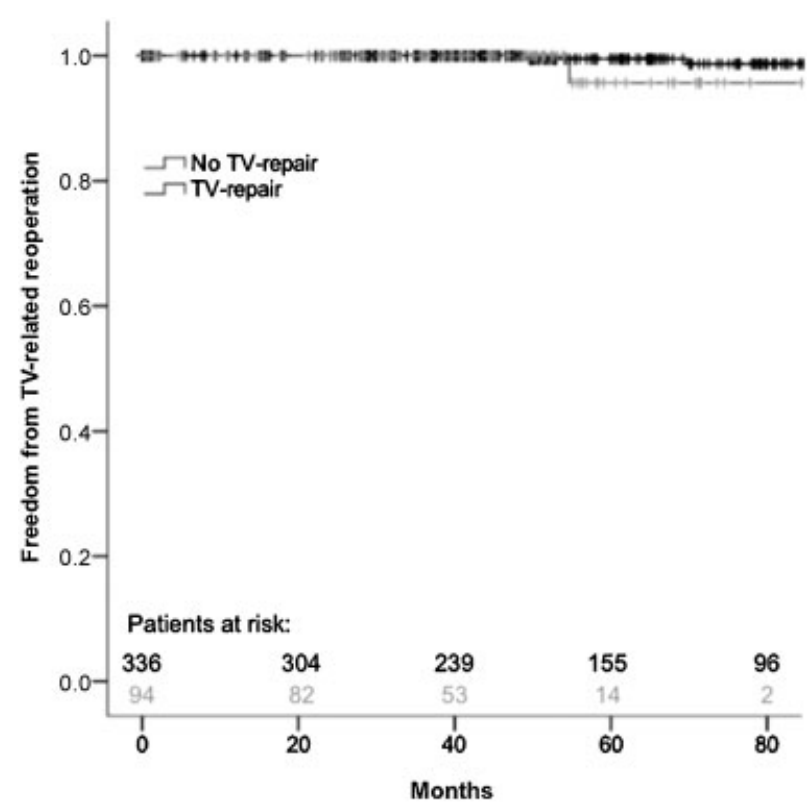

Fig. 5 Freedom from tricuspid valve (TV)-related reoperation for the two groups of patients.

( $p=0.8)$. TV-related reoperation had to be performed in four patients in group A between 21 days and 8.8 years postoperatively, and in one patient in group B 1.6 years postprocedure.

\section{Discussion}

We retrospectively assessed the implications of performing concomitant TV repair in patients with moderate functional TR undergoing elective minimal invasive MV surgery. Although concomitant TV surgery was associated with significantly longer cross-clamp, CPB, and operative times, we were unable to demonstrate any increased occurrence of perioperative complications in patients in whom additional TV repair was performed. In addition, we did not observe any significant difference in mid-term postoperative survival or freedom from TV-related reoperation between these two groups of patients. However, early postoperative rates of moderate or more residual TR were significantly higher in patients who did not undergo concomitant TV repair. In addition, residual $\mathrm{TR} \geq 2+$ was a predictor for poorer mid-term survival in the entire patient group.

One must be cautious when interpreting our results, as the observed lack of benefit for concomitant TV repair must be put in the perspective of probable patient selection bias. Our approach to moderate concomitant TR in MV patients was originally conservative in the early years of our study, but has become progressively more aggressive over time as more evidence supporting concomitant TV repair has appeared in the literature. Although all patients in the current study had moderate TR, it is quite likely that those who underwent concomitant TV repair had more advanced disease (e.g., increased TV annular dilation or right ventricular dilation and/or dysfunction). Indeed, patients who underwent concomitant TV repair had a higher incidence of atrial fibrillation, suggesting more advanced disease in this group. Despite this 
selection bias, the early and mid-term outcomes between the two groups of patients were similar. Combining this information with the observed decreased residual TR rate in concomitant TV repair patients, plus the observed decreased medium-term survival rate in patients with residual TR, one can conclude that concomitant TV repair should probably be performed in patients with moderate functional TR undergoing MV surgery.

Our conclusions are supported by the observations of several other investigators. In 2005, Dreyfus et al described an increase of the degree of TR of more than two grades during follow-up in $48 \%$ of patients undergoing MV surgery without initial concomitant TR. ${ }^{20}$ Matsunaga et al also observed an increase in TR after MV surgery due to functional ischemic mitral regurgitation. ${ }^{21}$ Both the authors stated that tricuspid annular dilatation is a criterion for the development of subsequent TR, regardless of the degree of initial TR. Dreyfus et al suggested that a dilated annulus of greater than $70 \mathrm{~mm}$ in the arrested heart or greater than $40 \mathrm{~mm}$ in the beating heart, regardless of the amount of TR, should be the criterion to perform additional TV repair in MV surgery patients.

Gender differences in patients undergoing TV repair with minimally invasive MV surgery for mitral regurgitation is another point of interest. Although there were no gender differences in patients undergoing concomitant TV repair for moderate TR, there was significant difference in patients who underwent concomitant TV repair (irrespective of TR grade), when the entire cohort of 2,183 patients was considered. In the latter group of patients, $14.7 \%$ were women and $8.1 \%$ were men $(p<0.001)$. This was similar to another report previously published by our institution ( $14.4 \%$ women vs. $8.2 \%$ men). ${ }^{22}$

Gender differences in patients undergoing TV repair with minimally invasive MV surgery for mitral regurgitation did not influence postoperative outcomes either, which matches the results of a previous study involving isolated TV surgery reported by us. We concluded: “... we were not able to find gender-dependent differences in the postoperative outcomes after isolated TV surgery. But there seem to be demographic gender-dependent differences regarding the indications for TV surgery, which could influence the treatment of these

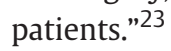

Other investigators have focused on the degree of TR at the time of MV surgery to address this issue. ${ }^{24,25}$ Calafiore et al performed a propensity score-adjusted analysis in 110 patients undergoing MV surgery due to functional MR with or without additional TV repair due to moderate or severe TR. These investigators found that patients undergoing additional TV repair had a significantly higher survival rate during follow-up (74.5\% vs. 45.0\%). Furthermore, they observed a benefit in clinical outcomes with $64.9 \%$ of TV repair patients being in NYHA class I to II during follow-up, compared with $35.2 \%$ in which the TV was not repaired. ${ }^{24}$ Kim et al investigated patients undergoing mechanical MV replacement due to rheumatic MV disease and additional TV repair in the presence of mild-to-moderate TR. ${ }^{26}$ They could show that there was no significant difference in the postoperative out- comes in patients with and without TV repair during a followup of 49 months. However, patients with TR grade $\geq 2+$ had a significantly worse survival compared with patients without TR. In a separate study, Navia et al investigated the postoperative outcome of patients undergoing left-sided heart valve surgery or coronary artery bypass grafting with or without additional TV repair in the presence of preoperative moderate $\mathrm{TR}^{25}$ They found no significant differences in the 5-year survival rate and TV-related reoperation rate between both the groups. However, postoperative echocardiographic measurements showed significantly less residual TR in the repair group that persisted during follow-up. In addition, they were able to demonstrate a clinical benefit with improved NYHA class in patients in whom the TV was repaired.

The use of intraoperative echocardiography to guide decision making regarding concomitant TV repair is not recommended because of the known difficulties calculating the degree of TR in sedated, ventilated patients. ${ }^{27}$ It is our current surgical strategy to perform additional TV repair if there is a dilated TV annulus of $\geq 40 \mathrm{~mm}$ as measured by preoperative echocardiography. ${ }^{20}$ Unfortunately, we were unable to retrospectively obtain consistent measurements of the preoperative tricuspid annular diameter in the current study, and therefore unable to comment on its effect on follow-up outcomes. It may be beneficial to develop a scoring system in the future that takes into account more information than just the tricuspid annular diameter because such a measurement may be operator dependent. Possible variables that could be incorporated into such a scoring system include vena contracta width, right atrial and pulmonary pressures, and evaluation of the presence of tricuspid structural abnormalities.

Current guidelines for additional treatment of moderate TR during MV surgery are based on expert opinions and retrospective studies. No prospective randomized studies currently exist, indicating the necessity to perform such a study to better clarify this important issue. Until such a study is performed, however, our current strategy is to follow the European Society of Cardiology (ESC) valvular guidelines and perform concomitant TV repair in patients with moderate functional TR and tricuspid annular dilation $>40 \mathrm{~mm}$ in patients undergoing MV surgery. ${ }^{11}$ Furthermore, we also perform concomitant TR in patients with annular dilation ( $>40 \mathrm{~mm}$ ) and less than moderate TR, as long as they do not have risk factors for complications due to prolonged $\mathrm{CPB}$ times. We believe that such a recommendation is prudent given the evidence accumulated from the studies cited above.

\section{Study Limitations}

The main limitation of the current study is its retrospective, non randomized nature. However, the current study represents one of the largest series to date of patients undergoing MV surgery with moderate TR and therefore may add further insights into this important issue. The other main limitation is our lack of detailed echocardiographic preoperative and follow-up data. This limitation is partially due to the fact that measurements of the tricuspid annulus were not routinely performed and recorded in the earlier 
years of the study, and that nearly one-third of our MV repair patients are referred from other regions of the country without the possibility to perform the follow-up data at our institution.

\section{Conclusion}

In conclusion, patients undergoing MV surgery with moderate functional TR do not experience increased perioperative complication rates when a concomitant TV repair is performed, despite longer operative times. Concomitant TV repair is associated with less residual TR early postoperatively, and residual TR is associated with worse survival during follow-up. Our observations, combined with those of other groups, support current recommendations to perform concomitant TV repair in such patients, particularly if tricuspid annular dilation is present.

\section{References}

1 Braunwald NS, Ross J Jr, Morrow AG. Conservative management of tricuspid regurgitation in patients undergoing mitral valve replacement. Circulation 1967;35(4, Suppl):I63-I69

2 Kwak JJ, Kim YJ, Kim MK, et al. Development of tricuspid regurgitation late after left-sided valve surgery: a single-center experience with long-term echocardiographic examinations. Am Heart J 2008;155(4):732-737

3 Song H, Kim MJ, Chung CH, et al. Factors associated with development of late significant tricuspid regurgitation after successful left-sided valve surgery. Heart 2009;95(11):931-936

4 Tang GH, David TE, Singh SK, Maganti MD, Armstrong S, Borger MA. Tricuspid valve repair with an annuloplasty ring results in improved long-term outcomes. Circulation 2006;114(1, Suppl): I577-I581

5 Van de Veire NR, Braun J, Delgado V, et al. Tricuspid annuloplasty prevents right ventricular dilatation and progression of tricuspid regurgitation in patients with tricuspid annular dilatation undergoing mitral valve repair. J Thorac Cardiovasc Surg 2011;141 (6):1431-1439

6 Bernal JM, Morales D, Revuelta C, Llorca J, Gutiérrez-Morlote J, Revuelta JM. Reoperations after tricuspid valve repair. J Thorac Cardiovasc Surg 2005;130(2):498-503

7 McCarthy PM, Bhudia SK, Rajeswaran J, et al. Tricuspid valve repair: durability and risk factors for failure. J Thorac Cardiovasc Surg 2004;127(3):674-685

8 Piñón M, Pinho P, Almeida J, Bastos P. Isolated surgery of tricuspid insufficiency in valvular reoperation. Rev Port Cir Cardiotorac Vasc 2005;12(1):11-14

9 Staab ME, Nishimura RA, Dearani JA. Isolated tricuspid valve surgery for severe tricuspid regurgitation following prior left heart valve surgery: analysis of outcome in 34 patients. J Heart Valve Dis 1999;8(5):567-574

10 Bonow RO, Carabello BA, Chatterjee K, et al; 2006 Writing Committee Members; American College of Cardiology/American Heart Association Task Force. 2008 Focused update incorporated into the ACC/AHA 2006 guidelines for the management of patients with valvular heart disease: a report of the American College of Cardiology/American Heart Association Task Force on Practice Guidelines (Writing Committee to Revise the 1998 Guidelines for the Management of Patients With Valvular Heart Disease): endorsed by the Society of Cardiovascular Anesthesiologists,
Society for Cardiovascular Angiography and Interventions, and Society of Thoracic Surgeons. Circulation 2008;118(15): e523-e661

11 Vahanian A, Baumgartner $\mathrm{H}$, Bax J, et al; Task Force on the Management of Valvular Hearth Disease of the European Society of Cardiology; ESC Committee for Practice Guidelines. Guidelines on the management of valvular heart disease: the Task Force on the Management of Valvular Heart Disease of the European Society of Cardiology. Eur Heart J 2007;28(2):230-268

12 Fehske W, Omran H, Manz M, Köhler J, Hagendorff A, Lüderitz B. Color-coded Doppler imaging of the vena contracta as a basis for quantification of pure mitral regurgitation. Am J Cardiol 1994;73 (4):268-274

13 Tribouilloy CM, Enriquez-Sarano M, Bailey KR, Tajik AJ, Seward JB. Quantification of tricuspid regurgitation by measuring the width of the vena contracta with Doppler color flow imaging: a clinical study. J Am Coll Cardiol 2000;36(2):472-478

14 Kuntze T, Borger MA, Falk V, et al. Early and mid-term results of mitral valve repair using premeasured Gore-Tex loops ('loop technique'). Eur J Cardiothorac Surg 2008;33(4):566-572

15 Seeburger J, Borger MA, Falk V, et al. Minimal invasive mitral valve repair for mitral regurgitation: results of 1339 consecutive patients. Eur J Cardiothorac Surg 2008;34(4):760-765

16 Mohr FW, Falk V, Diegeler A, Walther T, van Son JA, Autschbach R. Minimally invasive port-access mitral valve surgery. J Thorac Cardiovasc Surg 1998;115(3):567-574, discussion 574-576

17 Seeburger J, Borger MA, Passage J, et al. Minimally invasive isolated tricuspid valve surgery. J Heart Valve Dis 2010;19(2):189-192, discussion 193

18 Kay JH, Maselli-Campagna G, Tsuji KK. Surgical treatment of tricuspid insufficiency. Ann Surg 1965;162:53-58

19 Calkins H, Kuck KH, Cappato R, et al. 2012 HRS/EHRA/ECAS Expert Consensus Statement on Catheter and Surgical Ablation of Atrial Fibrillation: recommendations for patient selection, procedural techniques, patient management and follow-up, definitions, endpoints, and research trial design. Europace 2012;14(4): 528-606

20 Dreyfus GD, Corbi PJ, Chan KM, Bahrami T. Secondary tricuspid regurgitation or dilatation: which should be the criteria for surgical repair? Ann Thorac Surg 2005;79(1):127-132

21 Matsunaga A, Duran CM. Progression of tricuspid regurgitation after repaired functional ischemic mitral regurgitation. Circulation 2005;112;(9, Suppl):I453-I457

22 Seeburger J, Eifert S, Pfannmüller B, et al. Gender differences in mitral valve surgery. Thorac Cardiovasc Surg 2013;61(1):42-46

23 Pfannmueller B, Eifert S, Seeburger J, et al. Gender-dependent differences in patients undergoing tricuspid valve surgery. Thorac Cardiovasc Surg 2013;61(1):37-41

24 Calafiore AM, Gallina S, Iacò AL, et al. Mitral valve surgery for functional mitral regurgitation: should moderate-or-more tricuspid regurgitation be treated? A propensity score analysis. Ann Thorac Surg 2009;87(3):698-703

25 Navia JL, Brozzi NA, Klein AL, et al. Moderate tricuspid regurgitation with left-sided degenerative heart valve disease: to repair or not to repair? Ann Thorac Surg 2012;93(1):59-67, discussion 68-69

26 Kim JB, Yoo DG, Kim GS, et al. Mild-to-moderate functional tricuspid regurgitation in patients undergoing valve replacement for rheumatic mitral disease: the influence of tricuspid valve repair on clinical and echocardiographic outcomes. Heart 2012;98(1): 24-30

27 Jullien T, Valtier B, Hongnat JM, Dubourg O, Bourdarias JP, Jardin F. Incidence of tricuspid regurgitation and vena caval backward flow in mechanically ventilated patients. A color Doppler and contrast echocardiographic study. Chest 1995;107(2):488-493 